\title{
Control of Optical Waveguide-Resonator Coupling: Applications to Low-Power Optical Modulation and Switching
}

\author{
W. M. J. Green, R. K. Lee, A. Yariv, and A. Scherer \\ Applied Physics, California Institute of Technology, 1200 East California Blvd., Pasadena, CA 91125
}

Photonic integrated circuits employing microcavities coupled to optical waveguides have been studied extensively in recent years, for their ability to provide functionality important to optical telecommunications, within a compact form. Making use of the amplitude response imparted by the resonator upon the transmission spectrum of the coupled waveguide, passive channel add-drop filters [1] and polymer optical modulators based on electrooptic tuning of the ring resonator spectrum [2], have been demonstrated. For practical applications, it is desirable to engineer the drop channel/modulator OFF state extinction to be as large as possible. Recently, it was noted [3] that the waveguide-resonator coupling and the internal loss of the resonator are significantly related in the extinction performance of such devices. An optical modulator based on the principle of controlled waveguide-resonator coupling [4] has been designed to have large OFF state extinction. Furthermore, when compared to a conventional MachZehnder interferometer (MZI) modulator, the device presented here demonstrates significant reductions in the ON-OFF switching voltage, owing to the cavity enhanced phase response of the resonator.

The novel optical modulator geometry, depicted schematically in Figure 1, consists of a $2 \times 2$ MZI in which a feedback path is introduced by connecting an output waveguide to an input waveguide. The MZI functions as a voltage-controllable coupler to the ring resonator formed by the feedback path. Also shown in Figure 1 is an optical microscope image of the composite modulator fabricated in active InP/InGaAsP material. The InGaAsP waveguide core contains 6 quantum wells, tailored for optical gain near $\lambda=1.55 \mu \mathrm{m}$. The semiconductor device employs multimode interference (MMI) $3 \mathrm{~dB}$ couplers in the MZI, and the bend radius is $R=50 \mu \mathrm{m}$ in the curved sections of the racetrack-shaped resonator. Definition of waveguides and MMI couplers is accomplished by electron beam lithography. Channel waveguides are etched into the InP/InGaAsP material using ICP RIE with a $\mathrm{HI} / \mathrm{H}_{2} / \mathrm{Ar}$ plasma. Au electrical contacts are deposited on both arms of the MZI and on the racetrack resonator.

The optical output versus applied voltage can be characterized using equations (1)-(3). Quantities are defined as follows: $a_{1}\left(b_{1}\right)$ are the input(output) optical field amplitudes, $a_{2}$ and $b_{2}$ are the circulating fields in the ring, and $\alpha, \theta$ correspond to the transmission and phase shift per round trip in the ring, respectively. The relative phase shift $\Delta \phi$ in the MZI arms is written in terms of the MZI switching voltage, $V_{\pi}$

$$
\begin{gathered}
{\left[\begin{array}{l}
b_{1} \\
b_{2}
\end{array}\right]=i\left[\begin{array}{cc}
\cos \left(\frac{\Delta \phi}{2}\right)-\sin \left(\frac{\Delta \phi}{2}\right) \\
\sin \left(\frac{\Delta \phi}{2}\right) & \cos \left(\frac{\Delta \phi}{2}\right)
\end{array}\right]\left[\begin{array}{l}
a_{1} \\
a_{2}
\end{array}\right]=i\left[\begin{array}{c}
\cos \left(\frac{V}{2 V_{\pi}} \pi\right)-\sin \left(\frac{V}{2 V_{\pi}} \pi\right) \\
\sin \left(\frac{V}{2 V_{\pi}} \pi\right) \cos \left(\frac{V}{2 V_{\pi}} \pi\right)
\end{array}\right]\left[\begin{array}{l}
a_{1} \\
a_{2}
\end{array}\right]} \\
a_{2}=\alpha e^{i \theta} b_{2}
\end{gathered}
$$
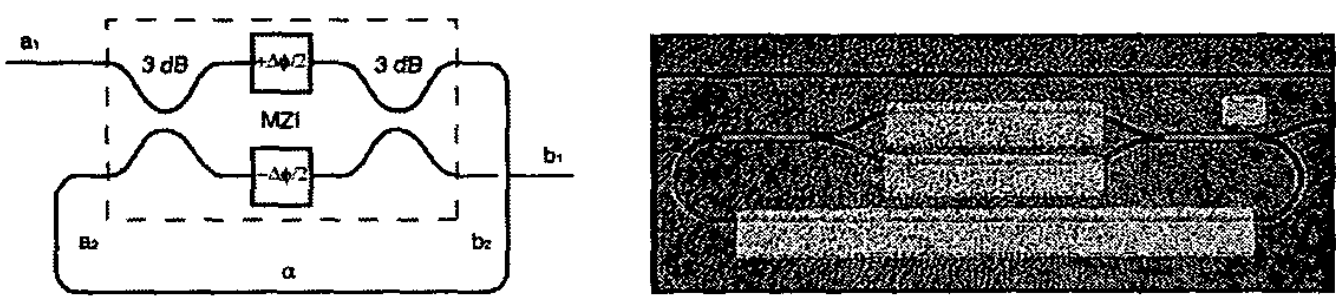

Figure 1: Left: Schematic of composite Mach-Zehnder-ring modulator. Right: InP/InGaAsP modulator. Total device length $\mathrm{L} \approx 800 \mu \mathrm{m}$. 
On resonance with the ring, $\theta=2 m \pi, m$ integer, the fraction of input power transmitted by the modulator is given by

$$
\frac{P_{\text {out }}}{P_{\text {in }}}=\left|\frac{b_{1}}{a_{1}}\right|^{2}=\frac{\left[\alpha-\left|\cos \left(\frac{V}{2 V_{\pi}} \pi\right)\right|\right]^{2}}{\left[1-\alpha\left|\cos \left(\frac{V}{2 V_{\pi}} \pi\right)\right|\right]^{2}} .
$$

The transmission of the composite MZI-ring modulator is plotted in Figure 2. For comparison, the typical $P_{o u} / P_{\text {in }}=\cos ^{2}\left[V \pi /\left(2 V_{\pi}\right)\right]$ transmission of a conventional MZI is plotted in the same figure. When the device is biased to the voltage giving minimum transmission, defined as $V_{c}$, the input optical power is completely dissipated by the losses of the ring resonator.

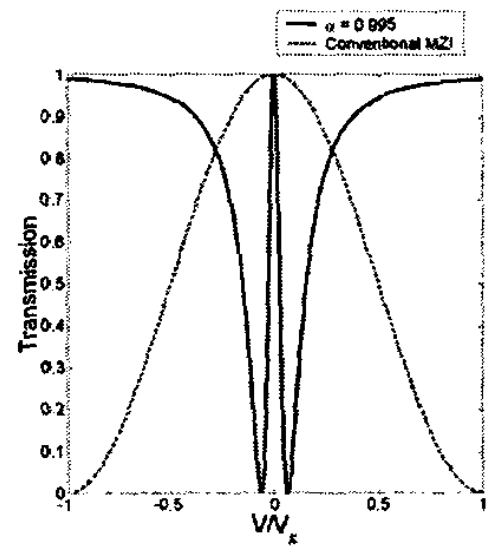

Figure 2: Solid curve: Composite modulator transmission, input tuned to resonance, $\alpha=0.995$. Dotted curve:

Conventional MZI modulator transmission.

Note that for a high-Q resonator $(\alpha \approx 1)$, the portion of the transmission curve near $V=0$ is very steep compared to the conventional MZI curve, resulting in a reduced switching voltage. The voltage reduction factor can be expressed as

$$
\frac{V_{c}}{V_{\pi}} \approx \sqrt{1-\alpha^{2}}
$$

For $\alpha=0.999, V_{d} / V_{\pi} \approx 1 / 32$, with an associated electrical power reduction factor of $P_{d} / P_{\pi} \approx$ 1/1024. Even in practical devices, where it may not be possible (or desirable) to achieve extremely low loss rings, the reduction in switching voltage for this modulator configuration can still be significant. The optical modulation bandwidth of this device is approximately equal to the inverse of the loaded resonator photon lifetime, which sets a practical limit on $\alpha$.

\section{Bibliography:}

[1] B. E. Little, J. S. Foresi, G. Steinmeyer, E. R. Thoen, S. T. Chu, H. A. Haus, E. P. Ippen, L. C. Kimerling, and W. Greene, IEEE Photon. Technol. Lett. 10, pp. 549-551, 1998.

[2] P. Rabiei, W. H. Steier, C. Zhang, L. R. Dalton, J. Lightwave Technol. 20, pp. 1968-1975, 2002.

[3] A. Yariv, Electron. Lett. 36, pp. 321-322, 2000.

[4] A. Yariv, IEEE Photon. Tech. Lett. 14, pp. 483-485, 2002. 